\title{
Short-term Evaluation of Negative Pressure Applied by the Multi-Pressure Dial System to Lower Nocturnal IOP: A Prospective, Controlled, Intra-subject Study
}

Jeffrey L. Goldberg • Jesus Jiminez-Roman • Alejandra Hernandez-Oteyza •

Hugo Quiroz-Mercado

Received: January 11, 2021 / Accepted: March 26, 2021 / Published online: April 19, 2021

(C) The Author(s) 2021

\section{ABSTRACT}

Introduction: The purpose of this study was to investigate the short-term safety and feasibility of negative pressure application by the MultiPressure Dial (MPD) System to lower nocturnal intraocular pressure (IOP) in subjects with openangle glaucoma (OAG).

Methods: A prospective, controlled, intra-subject study of 22 eyes from 11 subjects at a single site was performed. All subjects had a history of OAG and were currently using a topical prostaglandin. For each subject, the eye with the highest IOP in the supine position was selected as the treatment eye (TE) and the contralateral eye served as the control eye (CE). The negative pressure for the TE was set to $60 \%$ of the baseline IOP value with no negative pressure in the CE. IOP measurements were collected at three prespecified time points overnight in the supine position with active negative pressure. The primary outcome measure was mean IOP with the application of negative pressure.

Results: At the three overnight time points, the mean ( \pm standard deviation) baseline IOP prior to negative pressure application was $22.2 \pm 2.5 \mathrm{mmHg}$ in the TE and $21.8 \pm 2.5 \mathrm{mmHg}$ in the CE. With the application of $60 \%$ negative pressure to the TE and no active negative pressure to the $\mathrm{CE}$, the mean IOP was $14.2 \pm 2.2$ and $19.5 \pm 2.4 \mathrm{mmHg}$, respectively. The mean percentage IOP reduction in the TE was 35\% $(p<0.001)$. There were two minor adverse events, both unrelated to device wear, and there were no IOP spikes $\geq 10 \mathrm{mmHg}$.

Conclusion: The MPD can safely and effectively lower nocturnal IOP in the supine position. The MPD holds promise as a potential new, noninvasive treatment option for the control of nocturnal IOP.
J. L. Goldberg

Byers Eye Institute, Stanford University, Stanford, CA, USA

J. Jiminez-Roman $(\varangle) \cdot$ A. Hernandez-Oteyza Glaucoma Department, APEC, Mexico City, MX, USA

e-mail: jimenez_roman@yahoo.com

H. Quiroz-Mercado

Department of Ophthalmology, University of

Colorado School of Medicine, Aurora, CO, USA 
Keywords: Multi-pressure dial; Open-angle glaucoma; Normal-tension glaucoma; Nocturnal IOP; Multi-pressure glaucoma management

\section{Key Summary Points}

\section{Why carry out this study?}

The current glaucoma treatment landscape does not have a reliable treatment option for nocturnal intraocular pressure (IOP) control.

There is a trend towards elevated nocturnal IOP in glaucoma patients.

The IOP-lowering ability of the MultiPressure Dial (MPD) has not been evaluated at night.

\section{What was learned from the study?}

The MPD is capable of providing meaningful IOP reduction ( $>25 \%)$ in patients with glaucoma in the supine position at night.

The MPD is a non-invasive treatment option that may serve as an independent or adjunctive treatment option for nocturnal IOP control in the future.

\section{DIGITAL FEATURES}

This article is published with digital features, including a summary slide, to facilitate understanding of the article. To view digital features for this article go to https://doi.org/10.6084/ m9.figshare.14269835.

\section{INTRODUCTION}

Glaucoma remains the leading cause of irreversible blindness in the world and is expected to affect more than 100 million people by 2040 [1]. Current glaucoma treatments primarily target the reduction of intraocular pressure

(IOP), the only modifiable risk factor proven to be associated with disease progression [2, 3]. Over the last decade, several new surgical and medical treatment options have emerged [4-6], including the introduction of minimally invasive glaucoma surgery, a growing space of surgical treatment options that offer variable IOPlowering efficacy but are broadly purported to have a superior safety profile compared to traditional, filtering surgery options [4, 7]. Despite the expansion of treatment options, the current glaucoma treatment landscape remains devoid of a treatment option that can be categorized as non-laser, non-drug and non-invasive. Furthermore, treatment options for glaucoma are more limited for IOP lowering at night [8]. Recent studies have highlighted the importance of nocturnal IOP control for glaucomatous disease progression, particularly with respect to vulnerable patient subgroups, such as those with normal-tension glaucoma (NTG) [9, 10].

In this study we describe the use of the Multi-Pressure Dial system (MPD; Equinox Ophthalmic, Inc., CA, USA) to lower IOP. The MPD system consists of pressure-sensing goggles connected to a pressure-modulating pump that individually enclose each periorbital region. The principle of operation of the device is based on Pascal's law, which states that when there is a change in pressure at any point in a confined fluid, there is an equal change throughout the fluid. With a secure, air-tight fit and a negative pressure value programmed into the device, negative pressure (or vacuum) is applied to convey a localized decrease in atmospheric pressure contacting the surface of the eye. The localized decrease in pressure translates to a decrease in pressure inside the eye (IOP). After the goggles are appropriately fitted, target negative pressure is programmed via software into the pump and a target pressure value can be dialed in for each periorbital region, enabling the pressure reduction to be individualized for each eye with proper fit and wear of the device.

Prior studies have reported favorable safety and tolerability results for up to $8 \mathrm{~h}$ of continuous wear time with the MPD [11, 12]. However, the immediate IOP-lowering ability of the device at night has not been reported. Given the 
trend towards elevated nocturnal IOP in glaucoma patients, we sought to determine whether the MPD could address this unmet need in glaucoma management. This study aimed to evaluate the safety and feasibility of the MPD in lowering nocturnal IOP in the supine position.

\section{METHODS}

This prospective, fellow eye-controlled study was performed at a single site (Mexico City, Mexico). The study procedures were approved by Comité de Ética en Investigación, Universidad de Monterrey, the local Ethics Committee of the institution where the study was performed (reference number: 1208-2019-a-EXTCEI). The study procedures were in accordance with the 1964 Helsinki declaration and its later amendments or comparable ethical standard. All subjects included in the study provided informed consent prior to the beginning the study. This study was designated as a non-significant risk clinical study and did not require clinical trial registration.

Key inclusion criteria were age $\geq 22$ years, a documented diagnosis of open-angle glaucoma (OAG) in both eyes, periorbital anatomy that allowed a proper and secure, air-tight seal when the goggles were put in place and ability to tolerate IOP measurements with the device in place. Subjects who met the inclusion criteria had to have a screening IOP measurement of $\geq$ 16 and $\leq 32 \mathrm{mmHg}$ in both eyes and had to be currently using a prostaglandin analog medication for treatment of glaucoma in both eyes.

Exclusion criteria included the presence or history of any eye condition/disease that could compromise evaluation of the study results or subject safety. Subjects with macular degeneration, retinal detachment or other fundus findings that could prevent visualization of the retina in either eye, eyelid edema or conjunctival chemosis in either eye, history of corneal transplant in either eye, or history of allergy to any of the testing materials (e.g. silicone) were excluded. Subjects with a history of filtering glaucoma procedure (e.g. trabeculectomy, tube shunt) in either eye were also excluded, as were subjects with narrow anterior chamber anatomy
(Shaffer angle grade $\leq 2$ ) as visualized by gonioscopy. To limit variability in this small sample size, subjects currently using glaucoma medications other than prostaglandin analogs were also excluded.

\section{Study Materials}

The MPD primarily comprises two distinct components: pressure-sensing goggles and a programmable, pressure-controlling pump. The two components are connected with tubing that extends from the pump individually to each periorbital region. This design enables individualized negative pressure control for each eye.

A modified version of the MPD (only for investigational use), called the Excursion MPD, was used in this study at the study site to obtain IOP measurements at active negative pressure. The Excursion adaptation of the device contains an access port on each of the lenses that enables a pneumatonometer to make IOP measurements under active negative pressure. Because the negative pressure microenvironment requires a sealed environment, a specialized method of IOP measurement, also known as the excursion test method, was employed, as described in detail in [13]. Of note, the pneumatonometric measurement employed with the Excursion test method occurs across a latex membrane (tono-pen cover) and remains external to the negative pressure microenvironment. This method allows for IOP measurements relative to atmospheric pressure. A diagram of this method is shown in Fig. 1.

\section{Study Design}

During the baseline visit (visit 1) the subjects were: (1) assessed in terms of the inclusion/exclusion criteria; (2) checked that a proper seal could be achieved with the device; and asked to undergo a battery of baseline clinical tests, including best-corrected visual acuity, manifest refraction, IOP with Goldmann applanation tonometry (GAT), gonioscopy and comprehensive slit lamp and dilated fundoscopic examination. Additional IOP measurements were 


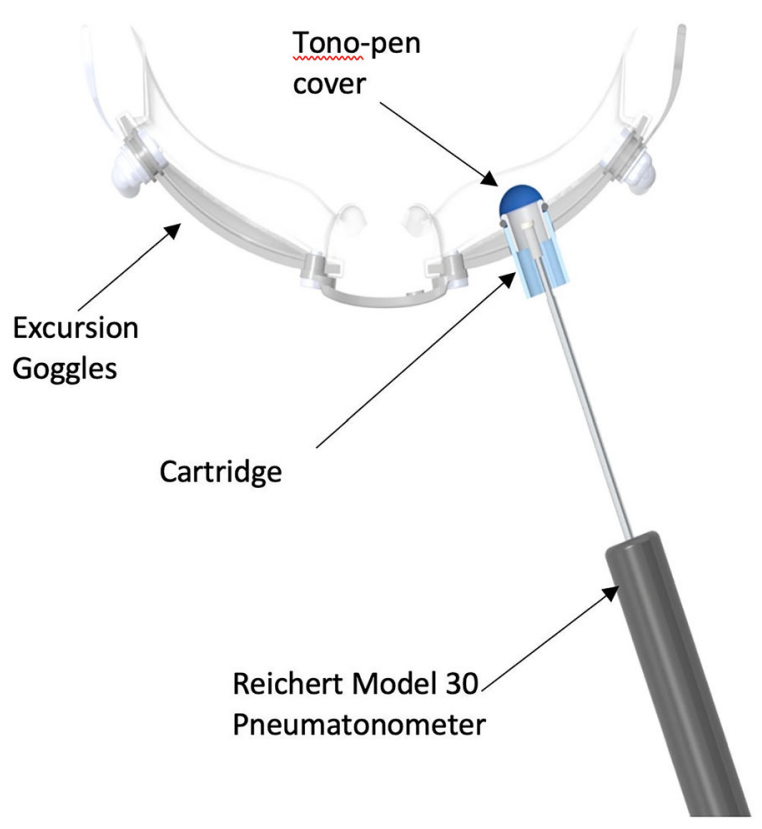

Fig. 1 The Excursion Multi-Pressure Dial system. The pneumatonometer is inserted through the open, distal end of the access port and gently placed against the tono-pen cover on the cornea to obtain an intraocular pressure measurement

obtained using the Reichert model 30 pneumatonometer (PTN; Reichert Inc., Buffalo, NY, USA) in both the upright (seated) and supine position. The eye with higher IOP based on supine pneumatonometry was assigned as the treatment eye (TE) while the intra-subject contralateral eye was assigned as the control eye (CE). Following assignment of the TE/CE, a sequence of IOP measurements were made in the supine position under various conditions. The initial measurements were obtained after the Excursion MPD was securely in place without negative pressure to establish a baseline IOP for both eyes. Prior to the application of negative pressure, the device was programmed with the appropriate negative pressure value for each eye. The negative pressure value for the TE was set to $60 \%$ of the supine IOP value measured by the PTN prior to the MPD being worn; for example, a subject with an IOP value of $20 \mathrm{mmHg}$ had a target negative pressure value set at $12 \mathrm{mmHg}$. After the MPD was securely in place with the correct target negative pressure setting, the IOP was obtained. This was done at visit 1 to ensure the IOP in the TE with active negative pressure was not harmful to the subject. A summary of the IOP measurements obtained at visit 1 are shown in Fig. 2.

Eligible subjects who met the inclusion criteria and completed the testing at visit 1 were scheduled to return for visit 2 , which consisted of an overnight stay, within 7 days of visit 1 . Visit 2 could occur as early as on the same day (evening) as visit 1 . At visit 2 , subjects were admitted to a sleep clinic. Each subject was refitted with the Excursion MPD, and three IOP

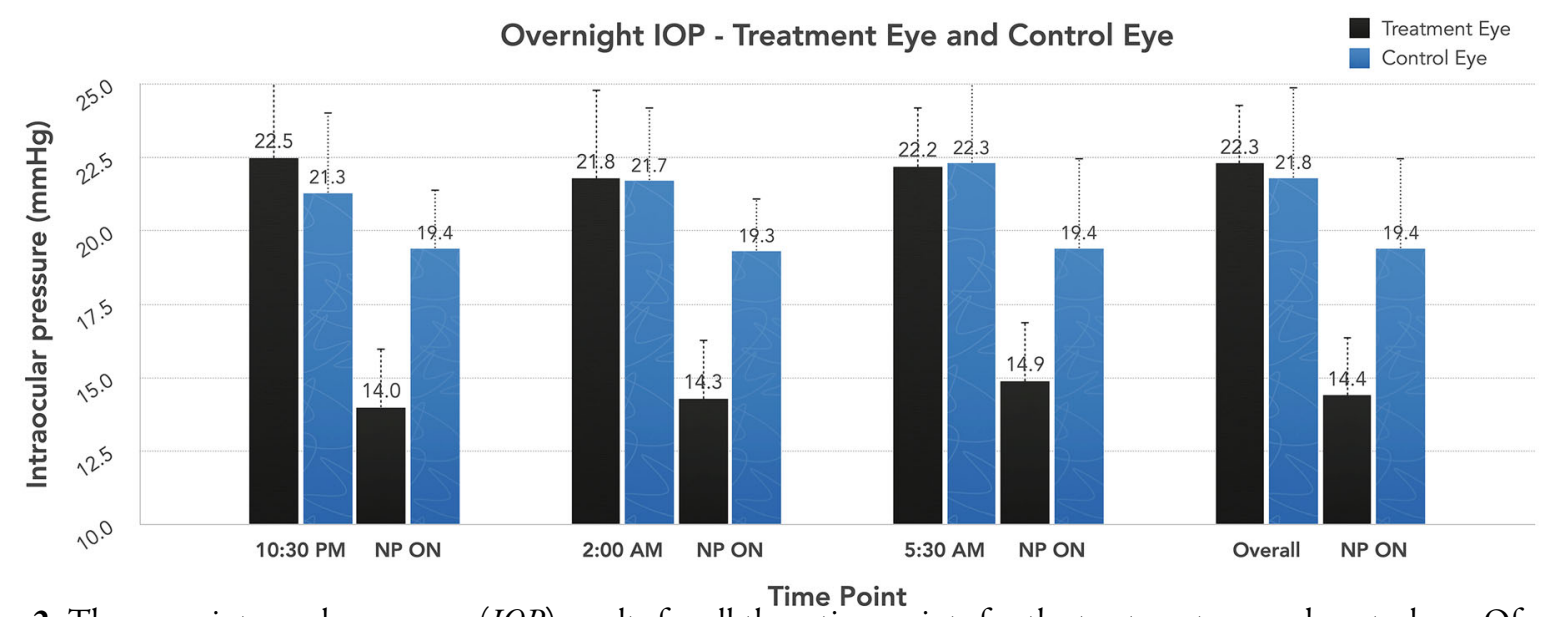

Fig. 2 The mean intraocular pressure $(I O P)$ results for all three time points for the treatment eye and control eye. Of note, the negative pressure $(N P)$ setting for the control eye was $0 \mathrm{mmHg}$. The far right group of bars shows the mean IOP across all three time points for the treatment eye and control eye 


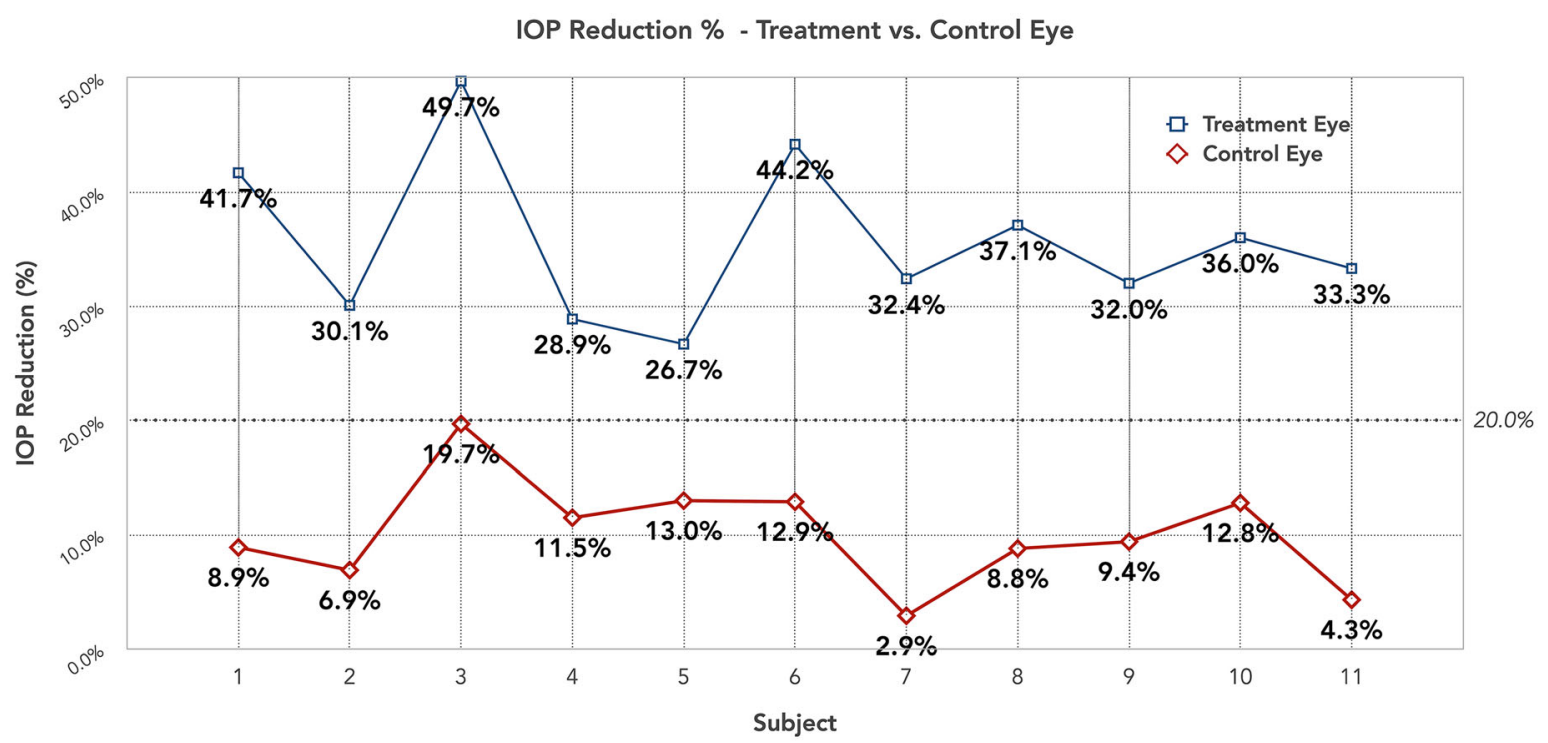

Fig. 3 Comparison of the percentage of IOP reduction in the treatment eye and control eye for each subject (subjects 1-11). It is noted that 9 of the 11 subjects achieved a $>30 \%$ IOP reduction in the treatment eye with the application of negative pressure

Following the three scheduled overnight IOP measurements, a repeat slit lamp examination was performed as well as repeat IOP measurements by GAT. In addition, an assessment of adverse events was performed.

\section{Outcome Measures}

The primary outcome measure in this study was the mean IOP with active negative pressure (as measured by PTN with the Excursion MPD in place) at the three prespecified time points during visit 2 . The mean IOP was evaluated for the study eye (TE) and CE separately at visit 2 .

The safety assessment included changes in slit lamp examination following visit 2 , the rate of ocular adverse events and the rate of IOP increases $\geq 10 \mathrm{mmHg}$ by GAT following visit 2 compared to the IOP measured at visit by GAT.

\section{Statistical Analysis}

A repeated-measures analysis of variance test was performed to evaluate the mean IOP with active negative pressure in the TE compared to baseline at the three overnight time points. Post 
hoc paired $t$ tests were performed to compare the mean IOP in the TE prior to and following the application of negative pressure with the baseline value at all three time points. Statistical analyses were performed using Prism 8 software (GraphPad Software Inc., San Diego, CA, USA). The significance level was set at $p \leq 0.05$.

\section{RESULTS}

\section{Subject Demographics}

A total of 11 subjects diagnosed with bilateral OAG were enrolled in and successfully completed the study. One subject did not adequately meet the inclusion criteria and was not included in the study. All subjects were Hispanic. The mean age of the subjects was $64.9 \pm 6.9$ years, and nine were women.

\section{IOP Results}

The IOP results for each time point in the overnight visit are shown in Fig. 1. At 10:30 p.m., the first overnight time point of visit 2 , the mean IOP in the TE and CE prior to the application of negative pressure was $22.5 \pm 3.2$ and $21.3 \pm 2.9 \mathrm{mmHg}$, respectively. Following the application of negative pressure to the TE, the mean IOP of the TE was $14.0 \pm 2.4 \mathrm{mmHg}$, or a $38 \%$ reduction $(p<0.001)$; the mean IOP of the $\mathrm{CE}$ at the same time point was $19.4 \pm 2.3 \mathrm{mmHg}$. At the second time point (2:00 a.m.), the mean IOP before negative pressure application was $21.8 \pm 2.22 \mathrm{mmHg}$ in the TE and $21.7 \pm 1.8 \mathrm{mmHg}$ in the CE. Following activation of negative pressure to the TE, the mean IOP in the TE was $14.3 \pm 2.3 \mathrm{mmHg}$, a $34 \%$ reduction $(p<0.001)$; the mean IOP in the control eye was $19.3 \pm 1.8 \mathrm{mmHg}$. At the third time point, 5:30 a.m., the mean IOP in the $\mathrm{TE}$ and CE prior to application of negative pressure was $22.2 \pm 2.2$ and $22.3 \pm 2.9 \mathrm{mmHg}$, respectively. After negative pressure application to the TE, the mean IOP in the TE was reduced by $35 \%$ to $14.4 \pm 2.1 \mathrm{mmHg}(p<0.001)$; in the $\mathrm{CE}$, at the same time point, the mean IOP was $19.9 \pm 3.0 \mathrm{mmHg}$.
Overall, across all three time points at night, the mean IOP prior to application of negative pressure was $22.2 \pm 2.5 \mathrm{mmHg}$ in the TE In the $\mathrm{CE}$, the mean IOP prior to negative pressure application was $21.8 \pm 2.5 \mathrm{mmHg}$. The mean IOP of the TE and CE groups prior to the application of negative pressure was not significantly different $(p=0.53)$. With the application of negative pressure in the TE and no application of active negative pressure in the $\mathrm{CE}$, the mean IOP was $14.2 \pm 2.2$ and $19.5 \pm 2.4 \mathrm{mmHg}$, respectively. Overall, the mean percentage IOP reduction in the TE with the application of negative pressure was 35\% $(p<0.001)$.

Figure 2 shows the comparison of IOP reduction between the TE and CE. Every subject achieved an IOP reduction of $>20 \%$ with negative pressure application in the TE at all three overnight time points. In $82 \%(9 / 11)$ of subjects, the mean IOP reduction was $\geq 30 \%$ across all three overnight time points.

\section{Safety Measures}

No qualitative changes in the TE or CE were observed on comprehensive slit lamp evaluation following the overnight study period at visit 2 . The mean IOP by GAT at visit 1 was $17.9 \pm 1.6 \mathrm{mmHg}$ in the right eye and $17.3 \pm 1.3 \mathrm{mmHg}$ in the left eye. Following visit 2 , repeat IOP measurements obtained in the upright position by GAT were $16.9 \pm 3.1$ and $16.3 \pm 2.1 \mathrm{mmHg}$ in the right and left eye, respectively. There were no instances of IOP increases $\geq 10 \mathrm{mmHg}$ in either the right or left eye measured bya GAT at visit 2 compared to baseline.

There were two adverse events that occurred during the study period. One subject had diarrhea during the study period which was selflimited and resolved without sequelae. Another subject had eye pain during the study period, and an examination revealed anterior uveitis for which the subject was treated with appropriate topical therapy. 


\section{DISCUSSION}

Clinicians managing patients with glaucoma almost always rely on IOP measurements obtained in-office, or during the day, to guide treatment decisions and assess successful management of disease progression. These measurements, however, only convey a brief snapshot of a patient's 24-h IOP profile and may consistently miss peak IOP levels [14]. Recent studies using 24-h IOP monitoring have demonstrated that for many patients, the peak IOP occurs at night [15-17]. Additionally, prior studies have demonstrated that glaucomatous patients endure larger fluctuations in IOP at night compared to healthy controls [18]. The current glaucoma treatment landscape would benefit from treatment options that safely lower IOP at night. Additionally, new adjunctive or independent treatments that provide nocturnal IOP control would address a sizable unmet need $[19,20]$.

This prospective, controlled study demonstrated the nocturnal IOP-lowering ability of the MPD device at three independent time points during the nocturnal period. At all three time points throughout the night, there was a statistically and clinically significant IOP reduction of $>30 \%$ from baseline while the device was worn with active negative pressure. The mean IOP in the TE was reduced to $<15 \mathrm{mmHg}$ at all three time points. This IOP reduction was observed in patients already on a topical prostaglandin, indicating the MPD can still achieve meaningful IOP reduction in eyes currently receiving medical treatment. Additionally, the IOP reduction was $>20 \%$ in all subjects regardless of baseline IOP (Fig. 2). Although negative pressure settings were not tailored to target goals for each patient in this study, the titratability of the MPD is an attractive element as some patients may be subject to larger increases in nocturnal IOP [21].

The safety results of this study were also favorable, corroborating prior studies evaluating the safety and tolerability of the device in healthy subjects $[11,12]$. There were no IOP spikes above baseline following the overnight visit in either the $\mathrm{CE}$ or TE. There were no changes observed on slit lamp examination in the anterior or posterior segment following visit 2 in comparison to baseline, with the exception of evidence of anterior inflammation detected in one patient that responded and resolved with appropriate topical therapy. Given the non-invasive nature of the device, it remains unlikely that its wearing prompted the development of anterior inflammation, in particular because this has not reported in any prior study evaluating the safety and efficacy of the device. Consistent with prior work evaluating the device, there were no serious adverse events.

A prior study investigating the safety and tolerability of continuous, nightly wear of the MPD over a 7-day period [22] produced favorable results, indicating that patients would be amenable to continuous overnight wear. This earlier study primarily focused on the safety and tolerability of the MPD and did not obtain IOP measurements in the nocturnal setting or supine position. Coupled with the results of this present study, these findings indicate the MPD could represent the first, non-invasive intervention for nocturnal IOP control and could be worn nightly as an adjunct or independent treatment option. The ability to safely and consistently control nocturnal IOP would be particularly meaningful for patients with NTG, a subset of glaucoma associated with greater fluctuations in nocturnal IOP and lower ocular perfusion pressure $[6,23]$.

Given the novelty of employing negative pressure to lower IOP, future investigation is critical. The findings of this study highlight the short-term reduction in nocturnal IOP, with an IOP reduction of $>20 \%$ at three different time points. It remains important, however, to note that the IOP reduction conferred by the MPD is limited to when the device is worn with negative pressure. With removal of the device, the IOP returns to baseline.

The limitations of this study include the small sample size, the single visit, the shortterm duration and the non-randomized design. Measuring IOP using a pneumatonometer and a tonometer tip cover remains a novel method of IOP measurement, and while favorable results regarding the accuracy and precision of the method have been reported in prior clinical 
work [13], no published benchtop studies have specifically investigated the precision of the method in a benchtop setting with active negative pressure. It remains unclear which effects, if any, the MPD may have over the long term with repeated wear at night for weeks to months. This study exclusively included Hispanic subjects, which limits the generalizability of this study to other patient populations. In this study, an IOP reduction was also observed in the contralateral, or control eye. Although a minor reduction, this has not been demonstrated in prior studies evaluating the IOP-lowering ability of the device $[22,24]$ and suggests future investigation is warranted evaluating the IOP-lowering ability of the MPD in the nocturnal setting and/or supine position.

\section{CONCLUSION}

Overall, the results of this short-term study are favorable, demonstrating that the MPD represents a non-invasive, safe option for reducing IOP in the supine position at night. This nonlaser, non-drug, non-invasive approach to nocturnal IOP control could be used adjunctively (as observed in this study) or as an independent treatment option. In conclusion, the significant reduction in IOP achieved through application of negative pressure to the periocular space indicates the MPD could fill an existing void for a non-invasive intervention that safely controls nocturnal IOP.

\section{ACKNOWLEDGMENTS}

We are grateful to the study participants for their participation in this study.

Authorship. All named authors meet the International Committee of Medical Journal Editors (ICMJE) criteria for authorship for this article, take responsibility for the integrity of the work as a whole, and have given their approval for this version to be published.

Author Contributions. All authors had complete access to the study data and assume full responsibility for the integrity of the data and the accuracy of the data analysis. AH-O, JJ-R and HQ-M contributed to the concept and design, data acquisition and data analysis/interpretation of this study. All authors assisted with drafting of the manuscript, critical revision of the manuscript and statistical analysis.

Disclosures. Jeffrey L Goldberg has received consulting fees from Equinox Ophthalmic, Inc. Jesus Jiminez-Roman, Alejandra HernandezOteyza and Hugo Quiroz-Mercado report no relevant financial disclosures.

Funding. This study was sponsored by Equinox Ophthalmic, Inc. (Newport Beach, CA). The journal's rapid service fee was funded by Equinox Ophthalmic, Inc.

Data Availability. The data set collected and analyzed for this present study is available from the corresponding author per reasonable request.

Compliance with Ethics Guidelines. The study procedures were approved by Comité de Ética en Investigación, Universidad de Monterrey, the local Ethics Committee of the institution where the study was performed (Mexico City, Mexico) (reference number: 1208-2019-aEXT-CEI). The study procedures were in accordance with the 1964 Helsinki declaration and its later amendments or comparable ethical standard. All subjects included in the study provided informed consent prior to the beginning the study. This study was designated as a non-significant risk clinical study and did not require clinical trial registration.

Open Access. This article is licensed under a Creative Commons Attribution-NonCommercial 4.0 International License, which permits any non-commercial use, sharing, adaptation, distribution and reproduction in any medium or format, as long as you give appropriate credit to the original author(s) and the source, provide a link to the Creative Commons licence, and indicate if changes were made. The images or other third party material in this article are included in the article's Creative Commons 
licence, unless indicated otherwise in a credit line to the material. If material is not included in the article's Creative Commons licence and your intended use is not permitted by statutory regulation or exceeds the permitted use, you will need to obtain permission directly from the copyright holder. To view a copy of this licence, visit http://creativecommons.org/licenses/bync/4.0/.

\section{REFERENCES}

1. Tham Y-C, Li X, Wong TY, Quigley HA, Aung T, Cheng C-Y. Global prevalence of glaucoma and projections of glaucoma burden through 2040: a systematic review and meta-analysis. Ophthalmology. 2014;121(11):2081-90. https://doi.org/10. 1016/j.ophtha.2014.05.013.

2. Heijl A, Leske MC, Bengtsson B, et al. Reduction of intraocular pressure and glaucoma progression: results from the early manifest glaucoma trial. Arch Ophthalmol. 2002;120(10):1268-79.

3. Leske MC, Hyman L, Hussein M, Heijl A, Bengtsson B. Comparison of glaucomatous progression between untreated patients with normal-tension glaucoma and patients with therapeutically reduced intraocular pressures. The effectiveness of intraocular pressure reduction in the treatment of normal-tension glaucoma. Am J Ophthalmol. 1999;127(5):625-6.

4. Shah M. Micro-invasive glaucoma surgery-an interventional glaucoma revolution. Eye Vis Lond Engl. 2019;6(1):29. https://doi.org/10.1186/s40662019-0154-1.

5. Kahook MY, Serle JB, Mah FS, et al. Long-term safety and ocular hypotensive efficacy evaluation of netarsudil ophthalmic solution: rho kinase elevated IOP treatment trial (ROCKET-2). Am J Ophthalmol. 2019;200:130-7. https://doi.org/10.1016/j.ajo. 2019.01.003.

6. Sheybani A, Scott R, Samuelson TW, et al. Openangle glaucoma: burden of illness, current therapies, and the management of nocturnal IOP variation. Ophthalmol Ther. 2020(1):1-14. https://doi. org/10.1007/s40123-019-00222-z.

7. Kotecha A, Feuer WJ, Barton K, et al. Quality of life in the tube versus trabeculectomy study. Am J Ophthalmol. 2017;176:228-35.
8. Orzalesi N, Rossetti L, Invernizzi T, Bottoli A, Autelitano A. Effect of timolol, latanoprost, and dorzolamide on circadian IOP in glaucoma or ocular hypertension. Invest Ophthalmol Vis Sci. 2000;41(9):2566-73.

9. Ciulla L, Moorthy M, Mathew S, et al. Circadian rhythm and glaucoma: what do we know? J Glaucoma. 2020;29(2):127-32.

10. De Moraes CG, Jasien JV, Simon-Zoula S, Liebmann JM, Ritch R. Visual field change and 24-hour IOPrelated profile with a contact lens sensor in treated glaucoma patients. Ophthalmology. 2016;123(4): 744-53. https://doi.org/10.1016/j.ophtha.2015.11. 020.

11. Thompson VM, Ferguson TJ, Ahmed IIK, et al. Short-term safety evaluation of a multi-pressure dial: a prospective, open-label non-randomized study. Ophthalmol Ther. 2019;82(11):887-9. https://doi.org/10.1007/s40123-019-0181-y.

12. Samuelson TW, Ferguson TJ, Radcliffe NM, et al. 8 hrs safety evaluation of a multi-pressure dial in eyes with glaucoma: prospective, open-label randomized study. Clin Ophthalmol Auckl NZ. 2019;13: 1947-53. https://doi.org/10.2147/OPTH.S217736.

13. Ferguson TJ, Knier CG, Chowdhury UR, et al. Intraocular pressure measurement with pneumatonometry and a tonometer tip cover. Ophthalmol Ther. 2020;9:127-37. https://doi.org/10. 1007/s40123-020-00235-z.

14. Quaranta L, Katsanos A, Riva I, et al. Twenty-fourhour intraocular pressure and ocular perfusion pressure characteristics in newly diagnosed patients with normal tension glaucoma. Eye Lond Engl. 2016;30(11):1481-9. https://doi.org/10.1038/eye. 2016.168.

15. Mosaed S, Liu JHK, Weinreb RN. Correlation between office and peak nocturnal intraocular pressures in healthy subjects and glaucoma patients. Am J Ophthalmol. 2005;139(2):320-4. https://doi.org/10.1016/j.ajo.2004.09.062.

16. Liu JHK, Mansouri K, Weinreb RN. Estimation of 24-hour intraocular pressure peak timing and variation using a contact lens sensor. PLoS ONE. 2015;10(6):e0129529. https://doi.org/10.1371/ journal.pone.0129529.

17. Aptel F, Musson C, Zhou T, Lesoin A, Chiquet C. 24-hour intraocular pressure rhythm in patients with untreated primary open angle glaucoma and effects of selective laser trabeculoplasty. J Glaucoma. 2017;26(3):272-7. https://doi.org/10.1097/ IJG.0000000000000604. 
18. Tojo N, Abe S, Ishida M, Yagou T, Hayashi A. The fluctuation of intraocular pressure measured by a contact lens sensor in normal-tension glaucoma patients and nonglaucoma subjects. J Glaucoma. 2017;26(3):195-200. https://doi.org/10.1097/IJG. 0000000000000517.

19. Liu JHK, Medeiros FA, Slight JR, Weinreb RN. Comparing diurnal and nocturnal effects of brinzolamide and timolol on intraocular pressure in patients receiving latanoprost monotherapy. Ophthalmology. 2009;116(3):449-54. https://doi.org/ 10.1016/j.ophtha.2008.09.054.

20. Liu JHK, Medeiros FA, Slight JR, Weinreb RN. Diurnal and nocturnal effects of brimonidine monotherapy on intraocular pressure. Ophthalmology. 2010;117(11):2075-9. https://doi.org/10. 1016/j.ophtha.2010.03.026.

21. Kim J, Caprioli J. Intraocular pressure fluctuation: is it important? J Ophthalmic Vis Res. 2018;13(2):170. https://doi.org/10.4103/jovr.jovr_35_18.
22. Ferguson TJ, Radcliffe NM, Van Tassel SH, et al. Overnight safety evaluation of a multi-pressure dial in eyes with glaucoma: prospective, open-label randomized study. Clin Ophthalmol. 2020;14: 2739-46. https://doi.org/10.2147/OPTH.S256891.

23. Bonomi L, Marchini G, Marraffa M, Bernardi P, Morbio R, Varotto A. Vascular risk factors for primary open angle glaucoma: the Egna-Neumarkt Study. Ophthalmology. 2000;107(7):1287-93. https://doi.org/10.1016/s0161-6420(00)00138-x.

24. Swan RJ, Ferguson TJ, Shah M, et al. Evaluation of the IOP-lowering effect of a multi-pressure dial at different negative pressure settings. Transl Vis Sci Technol. 2020;9(12):19. https://doi.org/10.1167/ tvst.9.12.19. 